\title{
Equations of motion method: Excitation energies and intensities in formaldehyde*
}

\author{
Danny L. Yeager ${ }^{\dagger}$ and Vincent McKoy $\neq$ \\ Arthur Amos Noyes Laboratory of Chemical Physics, ${ }^{\star}$ California Insitute of Technology, Pasadena. California 91109 \\ (Received 22 March 1973)
}

\begin{abstract}
We have used the equations of motion method to study the excitation energies and intensities of electronic transitions in formaldehyde. The calculated excitation energies and oscillator strengths agree well with experiment and suggest explanations for some unusual features recently observed in the optical absorption and electron scattering spectrum of formaldehyde in the vacuum ultraviolet.
\end{abstract}

\section{INTRODUCTION}

In recent papers ${ }^{1,2}$ we have discussed the equations of motion method as an approach for direct calculation of the properties of physical interest in spectroscopy, e.g., transition frequencies, intensities and scattering cross sections. By using a theory specifically designed for studying these relative properties one can avoid many of the difficulties involved in obtaining accurate values for absolute quantities such as the total energies. We have derived several approximations to the solution of the equations of motion for the operator $O_{\lambda}^{+}$which generates the state $|\lambda\rangle$ from the ground state $|0\rangle$. In the equations of motion method the approximation which includes single particle-hole $(1 p-1 h)$ and two particle-two hole $(2 p-2 h)$ components ${ }^{3}$ in $O_{\lambda}^{+}$, referred to as the $(1 p-1 h)+(2 p-2 h)$ approximation, gives results for transition frequencies and intensities in $\mathrm{N}_{2}, \mathrm{CO}, \mathrm{C}_{2} \mathrm{H}_{4},{ }^{2} \mathrm{C}_{6} \mathrm{H}_{6},{ }^{4}$ and $\mathrm{CO}_{2}{ }^{5}$ in good agreement with experiment.

In this paper we present results on the excited states of formaldehyde. These results include transition energies and oscillator strengths for transitions below $2000 \AA$ which allow us to explain some unusual features recently observed in the optical absorption ${ }^{6}$ and electron scattering spectrum. ${ }^{7}$ These studies were stimulated by the recent discovery of formaldehyde in interstellar dust clouds $^{8}$ and provided accurate absorption coefficients between 2400 and $912 \AA$. The absorption coefficients in the vacuum ultraviolet are large and hence absorption of photons in this spectral region is important in determining the fate of formaldehyde in the interstellar radiation field. Some of the unusual features in the spectrum that our results can explain include the prediction of a ${ }^{1} B_{1}\left(\sigma-\pi^{*}\right)$ state at $9.2 \mathrm{eV}$ with a very low oscillator strength and equal oscillator strengths for the transitions to the ${ }^{1} B_{2}\left(2 b_{2} 3 p a_{1}\right)$ and ${ }^{1} B_{2}\left(2 b_{2} 4 p a_{1}\right)$ states. The calculated $f$ value for each transition is 0.04 compared with the observed value of 0.03 . This distribution of intensities is unusual for a Rydberg series. Another interesting feature of the spectrum is the apparent absence of any band which could be related to the $\pi \rightarrow \pi^{*}$ valence transition. Our results show a transition to a ${ }^{1} A_{1}\left(\pi, \pi^{*}\right)$ state at $10.10 \mathrm{eV}$ with primarily intravalence character but with an $f$ value of only 0.10 . From the characteristics of this state there is a clearly an interaction between the neighboring ${ }^{1} A_{1}\left(2 b_{2} n p b_{2}\right)$ states and the $\pi^{*}$ valence state as discussed by Mentall et al. ${ }^{6}$

In the next section we give a very brief outline of our method. Section III discusses the results of our calcu- lations with different bas is sets designed to study specifif features in the observed spectrum. Section IV gives a summary of our conclusions.

\section{THEORY}

We have discussed the equations of motion method in recent papers ${ }^{1,3}$ and we will give only a very brief summary of the theory in this section. It can be shown that the operator $O_{\lambda}^{+}$which generates an excited state $|\lambda\rangle$ from the ground state, i. e., $|\lambda\rangle=O_{\lambda}^{+}|0\rangle$, is exactly a solution of the equation of motion ${ }^{9}$

$$
\left\langle 0\left|\left[\delta O_{\lambda}, H, O_{\lambda}^{+}\right]\right| 0\right\rangle=\omega_{\lambda}\left\langle 0\left|\left[\delta O_{\lambda}, O_{\lambda}^{+}\right]\right| 0\right\rangle,
$$

where $\delta O_{\lambda}^{+}$is a variation of the amplitudes specifying $O_{\lambda}^{+}$ and $\omega_{\lambda}$ the excitation frequency. The double commutator is defined as

$$
2[A, B, C]=[[A, B], C]+[A,[B, C]] .
$$

We have derived various approximations to the solution of $\mathrm{Eq}$. (1). ${ }^{1,3}$ If $O_{\lambda}^{+}$is restricted to single particle-hole $(1 p-1 h)$ form Eq. (1) becomes

$$
\left[\begin{array}{cc}
\mathbf{A} & \mathbf{B} \\
-\mathbf{B}^{*} & -\mathbf{A}^{*}
\end{array}\right]\left[\begin{array}{c}
\mathbf{Y}(\lambda) \\
\mathbf{Z}(\lambda)
\end{array}\right]=\omega_{\lambda}\left[\begin{array}{ll}
\mathbf{D} & 0 \\
O & \mathbf{D}
\end{array}\right]\left[\begin{array}{l}
\mathbf{Y}(\lambda) \\
\mathbf{Z}(\lambda)
\end{array}\right]
$$

where the elements of $\mathbf{A}, \mathbf{B}$, and $\mathrm{D}$ are defined in Ref. (1). We have also shown that the theory including $2 p-$ $2 h$ amplitudes in $O_{\lambda}^{+}$is equivalent to a renormalized $1 p-$ $1 h$ theory. We have derived an approximate perturbative scheme for solving these equations including $1 p-1 h$ and $2 p-2 h$ amplitudes. Unless we state otherwise the results of the equations of motion method will always refer to this approximation, i.e., $1 p-1 h+2 p-2 h$ approximation.

\section{RESULTS}

The first step in an equations of motion calculation is to carry out a self-consistent field calculation on the ground state of the molecule. These SCF orbitals form the particle-hole basis. The ground state electron configuration of formaldehyde is

$$
1 a_{1}^{2} 2 a_{1}^{2} 3 a_{1}^{2} 4 a_{1}^{2} 1 b_{2}^{2} 5 a_{1}^{2} 1 b_{1}^{2} 2 b_{2}^{2} .
$$

These calculations were done at the currently accepted ground state experimental geometry. ${ }^{10}$ In these calculations we used a $[3 s 2 p / 1 s]$ valence basis of contracted Gaussian functions ${ }^{2}$ to which we added diffuse Gaussian basis functions on the atomic centers and at the center of charge. We have found that such a basis can 
TABLE I. Excitation energies and intensities in formaldehyde. ${ }^{2}$

\begin{tabular}{|c|c|c|c|c|c|c|}
\hline State & $\begin{array}{l}\text { Main } \\
\text { transition }\end{array}$ & $\begin{array}{l}\Delta E^{\mathfrak{b}} \\
(\mathrm{EON})\end{array}$ & $\begin{array}{l}\Delta E \\
\text { (Obs.) }\end{array}$ & $\begin{array}{l}\Delta E \\
(\mathrm{CI})\end{array}$ & $f_{(\mathrm{EON})}$ & fobs. $)^{h}$ \\
\hline${ }^{3} A_{2}$ & $n \rightarrow \pi^{*}$ & 3.46 & $3.54^{c}$ & $3.41^{e}$ & & \\
\hline${ }^{1} A_{2}$ & $n \rightarrow \pi^{*}$ & 1.04 & $3.84^{\mathrm{c}}$ & 3.81 & & \\
\hline${ }^{3} A_{1}$ & $\tau \rightarrow \pi^{*}$ & 5.29 & $\cdots$ & 5.56 & & \\
\hline${ }^{1} A_{1}$ & $\pi \rightarrow \pi^{*}$ & 10.10 & $\ldots$ & $9.90^{\mathrm{f}}$ & 0.10 & \\
\hline${ }^{1} B_{1}$ & $\sigma \rightarrow \pi^{*}$ & 9.19 & $9.0^{\mathrm{d}}$ & 9.03 & 0.002 & \\
\hline${ }^{1} B_{2}$ & $n \rightarrow 3 s$ & 7.28 & 7.08 & 7.38 & 0.02 & 0.028 \\
\hline${ }^{i} B_{2}$ & $n \rightarrow 3 p a_{1}$ & 8.12 & 8.14 & 8.39 & 0.04 & 0.032 \\
\hline${ }^{1} A_{1}$ & $n \rightarrow 3 p b_{2}$ & 8.15 & 7.97 & 8.11 & 0.05 & 0.017 \\
\hline${ }^{1} A_{2}$ & $n \rightarrow 3 p b_{1}$ & 8.35 & $\ldots$ & $7.99^{8}$ & & \\
\hline${ }^{1} A_{1}$ & $n \rightarrow 4 p b_{2}$ & 9.40 & 9.58 & & 0.004 & $\ldots 1$ \\
\hline${ }^{1} A_{2}$ & $n \rightarrow 4 p b_{1}$ & 9.47 & $\cdots$ & & & \\
\hline${ }^{1} B_{2}$ & $n \rightarrow 4 p a_{1}$ & 9.55 & 9.63 & & 0.04 & 0.032 \\
\hline
\end{tabular}

${ }^{\text {a }}$ Vertical excitation energies.

${ }^{b}$ Results from the EOM method in the $(1 p-1 h)+(2 p-2 h)$ approximation. All energies in electron volts.

${ }^{c}$ See Ref. 11.

d The next six experimental values are from Ref. 6 .

${ }^{\circ} \mathrm{CI}$ calculations of Ref. 13.

${ }^{\mathbf{f}}$ Reference 14.

${ }^{\mathrm{g}}$ Reference 15.

${ }^{\mathrm{h}}$ Reference 7 .

'Observed to be much weaker than the transition to the

${ }^{1} B_{2}\left(2 b_{2} 4 p a_{1}\right)$.

adequately describe the intravalence transitions and also transitions to the first members of the Rydberg series. The basis must also contain these Rydberg components so as to study the valence-Rydberg mixing probably responsible for some features in the formaldehyde spectrum. Since the equations of motion method involves commutators that lead to operators of low rank, we can expect a basis with a s mall number of valence components to be adequate for carrying out calculations on most excited states including intravalence transitions. The diffuse components of our basis includes an $s$ function with an exponent of 0.05 on each atomic center, a $p$ function with an exponent of 0.05 on the carbon and oxygen centers, an $s$ function and a set of $p$ functions with an exponent of 0.016 at the center of charge, and also a $p_{x}$ and $p_{y}$ function with an exponent of 0.005 at the center of charge. In these calculations we truncated the particle basis slightly so as to use available computer programs. The resulting matrices were no larger than of order $30 \times 30$.

Table I shows the excitation energies for twelve transitions in formaldehyde along with the oscillator strengths for the seven dipole-allowed transitions. The calculated results listed in the third column agree well with the observed values in the fourth column. The experimental values for transitions to the ${ }^{1} A_{2}\left(n \rightarrow \pi^{*}\right)$ and ${ }^{3} A_{2}\left(n \rightarrow \pi^{*}\right)$ states are the estimated vertical excitation energies. ${ }^{11}$ The ${ }^{1} A_{1} \rightarrow{ }^{3} A_{1}\left(\pi \rightarrow \pi^{*}\right)$ has not been observed experimentally but could probably be seen quite easily in low-energy high-angle electron scattering off formaldehyde. The calculated excitation energy of $9.2 \mathrm{eV}$ for the ${ }^{1} A_{1} \rightarrow{ }^{1} B_{1}\left(\sigma \rightarrow \pi^{*}\right)$ transition is close to the value of $9 \mathrm{eV}$ inferred from the observed spectrum by Mentall et al. ${ }^{6}$ The oscillator strength of this transition is only 0.002 in agreement with the assignment of weak absorption features between 1340 and $1430 \stackrel{\mathrm{A}}{(}(8.7-9.4 \mathrm{eV})$ to the ${ }^{1} B_{1}\left(\sigma \rightarrow \pi^{*}\right)$ state. This is the only intravalence transition assigned in the observed spectrum below $2000 \AA$.

The ${ }^{1} A_{1}\left(\pi \rightarrow \pi^{*}\right)$ state is one of the puzzling aspects in the interpretation of the spectrum of formaldehyde. No feature was observed in either the absorption ${ }^{6}$ or electron scattering ${ }^{7}$ spectrum which could be related to the $\pi \rightarrow \pi^{*}$ excitation. We predict an excitation energy of $10.1 \mathrm{eV}$ and an $f$ value of 0.1 for this transition. This state is a valence-like state with some admixture of Rydberg character through its interaction with nearby Rydberg states. ${ }^{6}$ For example the change in the average value of $\sum_{i}\left(x_{i}^{2}+y_{i}^{2}\right)$ for the transition is about 15 $(\text { a. u. })^{2}$ which is larger than typical values for pure intravalence transitions, i.e., 2-3 (a.u. $)^{2}$ but much smaller than the values of 20-50 (a.u. $)^{2}$ which characterize the first members of Rydberg series. The $f$ value of 0.1 for this transition is clearly brought about by the mixing of the intravalence $\left(\pi \rightarrow \pi^{*}\right)$ state and the ${ }^{1} A_{1}$ Rydberg states with the configurations $\left(2 b_{2} n b_{2}\right)$ and $\left(1 b_{1} n b_{1}\right)$ in the present calculation. This basis does not allow for the mixing of ${ }^{1} A_{1}\left(2 b_{2} n d b_{2}\right)$ states since it does not contain $n d b_{2}$ functions. From the model calculations of Mentall et al. ${ }^{6}$ we can expect strong mixing between the $\pi \rightarrow \pi^{*}$ state and the ${ }^{1} A_{1}\left(2 b_{2} n d b_{2}\right)$ states. In the valence $[3 s 2 p / 1 s]$ basis ${ }^{2}$ the ${ }^{1} A\left(\pi \rightarrow \pi^{*}\right)$ state has a large $f$ value of about 0.4 which decreases due to Rydberg-valence mixing. If the final $f$ value for this transition does remain close to 0.1 we will not expect to see a strong feature in the absorption since this intensity will be spread over the entire band which can easily be $1 \mathrm{eV}$ wide. Our results show a ${ }^{1} A_{1}$ state near the $2 b_{2}$ ionization potential which has a large $f$ value of 0.2 . This may be an important feature in the spectrum but our present results are insufficient to identify it reliably. We are now carrying out more extensive calculations including the ${ }^{1} A_{1}\left(2 b_{2} n d b_{2}\right)$ states in order to understand the intensity distribution in the $8-12 \mathrm{eV}$ region. This includes a study of the generalized oscillator strength as a function of the square of the momentum transfer. The presence and position of minima in these oscillator strengths as a function of $q^{2}$ should clarify many of these questions.

The other results in Table I are all for Rydberg transitions of the type $2 b_{2} \rightarrow n s$ and $2 b_{2}-n p$. The excitation energies agree well with experiment and the $f$ values explain some interesting features in the spectrum. The ${ }^{1} B_{2}\left(2 b_{2} 3 p a_{1}\right)$ and ${ }^{1} B_{2}\left(2 b_{2} 4 p a_{1}\right)$ states have observed $f$ values of 0.032 . This is an unusual distribution of intensities for two successive members of a Rydberg series. The calculated $f$ value of 0.04 for both transi tions reproduces the observed distribution of intensity. These $f$ values are also far greater than the values given by the single-configuration approximation. ${ }^{6}$ The observed term value of $2.9 \mathrm{eV}$ for the ${ }^{1} A_{1}\left(n-3 p b_{2}\right)$ state is larger than the corresponding term value in typical $n p$ series, e.g., around $2.4 \mathrm{eV}$. This is again probably due to the perturbation of this state by the ${ }^{1} A_{1}\left(\pi \rightarrow \pi^{*}\right)$ state. The calculated $f$ value of 0.05 for the ${ }^{1} A_{1}(n$ $-3 p b_{2}$ ) state is larger than the observed value of 0.017 but here we can expect some redistribution of $f$ values when ${ }^{1} A_{1}\left(2 b_{2} n d b_{2}\right)$ states are included. This mixing of 
the ${ }^{1} A_{1}\left(2 b_{2} n d b_{2}\right)$ states can also put the ${ }^{1} A_{1}\left(2 b_{2} 3 p b_{2}\right)$ state below the ${ }^{1} B_{2}\left(2 b_{2} 3 p a_{1}\right)$. No experimental $f$ value was reported for the ${ }^{1} A_{1}\left(2 b_{2} 4 p b_{2}\right)$ state but a visual esti mate of the absorption coefficients shows that the transition to this state is much less intense than the ${ }^{1} A_{1}$ $-{ }^{1} B_{2}\left(2 b_{2} 4 p a_{1}\right)$ transition. The calculated $f$ values agree with this trend. We have also calculated the excitation frequencies to the triplet Rydberg states but these results are not listed in Table I since they are usually within $0.3 \mathrm{eV}$ of the corresponding singlet excitation energies.

We also obtain excitation energies and intensities of transitions to Rydberg states leading to the second ionization potential. These include the ${ }^{1} B_{1}(\pi-3 s)$ and ${ }^{1} B_{1}\left(\pi \rightarrow 3 p a_{1}\right)$ states at 11.2 and $12.2 \mathrm{eV}$ with $f$ values of 0.06 and 0.02 , respectively.

In the fifth column of Table I we list the excitation energies for many of these transitions obtained by the configuration interaction (CI) method. Many of these are results from extensive $\mathrm{CI}$ studies involving matrices ranging in order from $150 \times 150^{12}$ to $450 \times 450 .{ }^{13}$ The results agree well with the observed values and with those obtained from the equations of motion method. It is not our purpose to compare the conceptual or computational differences between these two methods. Excitation frequencies and intensities are just two quantities among others which the EOM method is designed to obtain. Other properties include cross sections for processes such as photoionization and electron molecule scattering. ${ }^{5}$

An interpretation of the formaldehyde spectrum in the vacuum ultraviolet requires an understanding of the ${ }^{1} A(\pi$ - $\pi^{*}$ ) state and its perturbation by the adjacent Rydberg states. For this reason we have carried out some additional calculations on this state and others with a different atomic basis. Configuration interaction calculations had previously placed the ${ }^{1} A\left(\pi \rightarrow \pi^{*}\right)$ state at around $11.4 \mathrm{eV}^{12,14}$ with an $f$ value of $0.4 .^{12}$ However, Whitten ${ }^{13}$ recently obtained a vertical excitation energy of $9.90 \mathrm{eV}$ in a very extensive CI calculation involving 468 configurations. This lowering relative to his previous result ${ }^{14}$ is partly due to the inclusion of $d$-type polarization functions in the basis and, more importantly, to an effective inclusion of sigma-pi correlation. To study the behavior of this state in a more flexible valence atomic basis we have done two additional calculations. In the first calculation we used a larger valence $[4 s 3 p / 2 s]$ basis of contracted Gaussian functions ${ }^{15}$ and for the second calculation this basis was augmented by $x z, y z$, and $z^{2}$ $d$-type polarization functions and diffuse $s$ and $p_{x}$ functions on the carbon and oxygen centers. The basis of the second calculation has both a larger valence component and the flexibility to reflect valence-Rydberg mixing. We obtained excitation energies of 9.90 and $9.66 \mathrm{eV}$ from the first and second calculation, respectively. The $f$ values are both close to 0.10 . These results again indicate the presence of an intravalence ${ }^{1} A_{1}\left(\pi-\pi^{*}\right)$ state around $9.9 \mathrm{eV}$.

\section{CONCLUSIONS}

We have used the equations of motion method to study the excitation energies and intensities of formaldehyde in the vacuum ultraviolet. This region of the formaldehyde spectrum has only recently been studied experimentally and shows some unusual features. ${ }^{6}$ The calculated transition energies and intenisties agree well with the observed values and suggest explanations for some of the unusual behavior in the spectrum. In agreement with experiment we see that intensities in the ${ }^{1} A_{1}\left(2 b_{2} n p b_{2}\right)$ series are normal but very unusual in the ${ }^{1} B_{2}\left(2 b_{2} n p a_{1}\right)$ series. Another interesting conclusion is the location of a ${ }^{1} A_{1}\left(\pi \rightarrow \pi^{*}\right)$ state at around $9.9 \mathrm{eV}$ but with the low $f$ value of 0.1 . This state is being perturbed by valence-Rydberg mixing. The generalized oscillator strength as a function of $q^{2}$ will be useful in studying these perturbations quantitatively.

${ }^{*}$ Research supported by the National Science Foundation.

$\dagger$ NSF Predoctoral Fellow 1970-1973.

Aifred P. Sloan Fellow.

$\S$ Contribution No. 4662 .

${ }^{1}$ T. Shibuya and V. McKoy, Phys. Rev. A 2, 2208 (1970).

${ }^{2} \mathrm{~J}$. Rose, T. Shibuya, and V. McKoy, J. Chem. Phys. 58, 74 (1973).

${ }^{3}$ T. Shibuya, J. Rose, and V. McKoy, J. Chem. Phys. 58, 500 (1973).

${ }^{4}$ J. Rose, T. Shibuya, and V. McKoy, J. Chem. Phys, 60, 2700 (1974).

${ }^{5} \mathrm{C}$. W. McCurdy and V. McKoy, "Differential cross sections for the scattering of electrons by $\mathrm{CO}_{2}$," (to be published).

${ }^{6}$ J. E. Mentall, E. P. Gentieu, M. Krauss, and D. Neumann, J. Chem. Phys. 55, 547 (1971).

${ }^{7}$ M. J. Weiss, C. E. Kuyatt, and S. Mielczarek, J. Chem. Phys. 54, 4147 (1971).

${ }^{8}$ L. E. Snyder, D. Buhl, B. Zuckerman, and P. Palmer, Phys. Rev. Lett. 22, 679 (1969).

${ }^{9}$ D. J. Rowe, Rev. Mod. Phys. 40, 153 (1968).

${ }^{10} \mathrm{~T}$. Oka, J. Phys. Soc. (Japan) 15, $2274(1960)$

${ }^{11}$ Low energy electron impact results of A. Chutjian Jet Propulsion Laboratory, Pasadena, CA (unpublished).

${ }^{12} \mathrm{~S}$. D. Peyerimhoff, R. J. Buenker, W. E. Kammer, and H. Hsu, Chem. Phys. Letters 8, 129 (1971).

${ }^{13}$ J. L. Whitten, J. Chem. Phys. 56, 5458 (1972).

${ }^{14} \mathrm{~J}$. L. Whitten and M. Hackmeyer, J. Chem. Phys. 51, 5584 (1969).

${ }^{15}$ For details of this basis set see T. H. Dunning, Jr., J. Chem. Phys. 53, 2823 (1970). 